\title{
New Micronutrient Fertilizer Biocomponents Based on Seaweed Biomass
}

\author{
Lukasz Tuhy, Mateusz Samoraj*, Sylwia Baśladyńska, Katarzyna Chojnacka \\ Department of Advanced Material Technologies, Faculty of Chemistry, Wroclaw University of Technology, \\ Smoluchowskiego 25, 50-372 Wroclaw, Poland \\ Received: January 28, 2015 \\ Accepted: April 1, 2015
}

\begin{abstract}
In the present paper, the novel environmentally friendly micronutrient fertilizer biocomponents produced via biosorption and based on the biomass of seaweed were introduced. The utilitarian properties of new preparations were examined in germination tests on Lepidium sativum. The application of micronutrient biocomponents with zinc resulted in the higher mass of plant and higher content of zinc in comparison with the untreated group and reference materials, leading to the biofortification of plants with this micronutrient (a more than 20-fold increase in zinc level was obtained). It was observed that the dosage of zinc influenced plant mass and element content of plants. Statistically significant correlations between zinc and other microand macronutrients were observed. Also, a strong positive correlation ( 0.55$)$ between zinc and plant mass was found.

The micronutrient biocomponents produced via the biosorption process can constitute an alternative tool for micronutrient fertilization as preparations characterized by high bioavailability of nutrients and high biodegradability constituting an "eco-friendly" method for micronutrient biofortification of plants.
\end{abstract}

Keywords: waste biomass, biosorption, zinc, micronutrient fertilizers, bioavailability

\section{Introduction}

The role of micronutrients is crucial for living organisms. Their participation in proper functioning of various biochemical pathways was proved. There are some notifications describing micronutrient deficiency in soils and plants, and hence in humans as a problem greater than hunger [1]. Micronutrient malnutrition was indicated as the main reason for serious health problems and developmental disorders [2]. From all micronutrients, zinc deficiency contributes to a higher extent to mortality [3]. Zinc is an essential micronutrient due to its important role in crucial biochemical processes in living organisms [4]. The availability is mainly affected by low content of the micronutrient in soils, high soil $\mathrm{pH}$, high salt concentration, and high

*e-mail: mateusz.samoraj@pwr.edu.pl phosphate content [5]. Zinc deficiency was observed also due to modern agriculture and inappropriate application of fertilizers [6].

Biofortification of plants with micronutrients is currently presented as the main and most efficient tool for combating micronutrient deficiency [7]. It constitutes the most effective strategy for micronutrients delivery [8]. There are two ways of plant biofortification: with the use of genetic engineering or via micronutrient fertilization. Genetic biofortification is a cost-effective solution, although the regulations on genetically modified food are still very restrictive, which can hamper its implementation [9]. Agronomic biofortification is a more accepted tool and its implementation readiness is higher [10]. Currently, agronomic biofortification is considered the most efficient way to combat micronutrient malnutrition [11]. Despite the very important role of micronutrients and their influence on plant condition 
and human health, still relatively low attention is put on innovations in micronutrient fertilization [12]. Due to some disadvantages of the application of traditional micronutrient fertilizers (eutrophication, toxicity), there is a need for new innovative preparations that are cheap, biodegradable, and characterized by high bioavailability of nutrients [13].

Biosorption was described as the ability of the surface of non-living biomass to bind and concentrate metal cations [14]. The biosorption was widely used in wastewater treatment for the purification of water solutions from toxic metals [15]. Different materials were used as biosorbents while macroalgae and microalgae were shown to be characterized by the best sorption properties $[16,17]$. Research on the use of the biosorption process in animal feeding currently is underway. The use of biomass of algae enriched with micronutrient ions as an alternative for traditional micronutrient products (inorganic salts and cheaters). It was shown that the application of micronutrient feed additives instead of inorganic salts and chelates - led to the biofortification of animal meat, milk, or eggs with micronutrients $[18,19]$. There is also some research describing the application of different types of biomass (peat, compost, berries, seeds) enriched with micronutrients as potential micronutrient biocomponents [20,21].

The aim of the present work was to examine the possible application of algae biomass enriched with zinc ions in the biofortification of Lepidium sativum in comparison with commonly used inorganic salts and chelates.

\section{Experimental Procedures}

\section{Sample Preparation}

For the biosorption experiments, three types of algae biomass were used: macroalgae Fucus sp. (Fucus), Baltic seaweed (Seaweeds) collected from the Baltic coast during summertime, and postextraction residues (Residues) after supercritical $\mathrm{CO}_{2}$ extraction conducted on these seaweeds. The biosorption of zinc(II) ions by biological materials was conducted in batch mode in stirred tank reactors $(60 \mathrm{~L})$. The concentration of $\mathrm{Zn}$ (II) ions (as $\mathrm{ZnSO}_{4} \cdot 7 \mathrm{H}_{2} \mathrm{O}$ (Chempur, Poland)) in the solution was $300 \mathrm{mg} / \mathrm{L}$, pH 5 measured in $25^{\circ} \mathrm{C}$ with the use of a Mettler Toledo SevenMulti $\mathrm{pH}$ meter, Switzerland. The concentration of the biomass was $1.0 \mathrm{~g}$ of dry mass (DM)/L according to our previous studies [22]. After the experiment, the solution was then filtered and the biomass was air-dried. The content of elements in the enriched biomass was examined by ICP-OES.

\section{Germination Tests}

The aim of experiments was to evaluate the effect of different types of algae biomass enriched with $\mathrm{Zn}$ (II) ions in the biosorption process on the germination of seeds and element content of germinated plants, when compared with the control groups of deionized water, cheated, and inorganic salt. Doses of $\mathrm{Zn}$ (II) used in the experiment varied from 1.0 to $6.0 \mathrm{mg} /$ Petri dish (four different doses) for each fertilizer. On this basis, the optimal dose of $\mathrm{Zn}(\mathrm{II})$ in the fertilizer was chosen. Five types of fertilizer components were used: macroalgae enriched with $\mathrm{Zn}$ (II) ions - Baltic seaweed (Baltic Seaweed-Zn), post-extraction residues (Residues-Zn), Fucus sp. (Fucus-Zn), Zn-EDTA chelate (Symposia Zn, Anwil, Poland), and $\mathrm{ZnSO}_{4} \cdot 7 \mathrm{H}_{2} \mathrm{O}$ (Chempur, Poland). To compare the fertilizer properties, germination tests were performed on garden cress Lepidium sativum according to the international norm (the International Seed Testing Association). Plastic Petri dishes covered with cotton (approximately $5.0 \mathrm{~g}$ ) soaked with deionized water were prepared. On each dish, 50 seeds of garden cress were placed in rows at equal distances from each other. In the next step seeds were subjected to stratification at $1^{\circ} \mathrm{C}$ for three days. After stratification, appropriate amounts of particular fertilizer were spread evenly on Petri dishes. Each probe was taken in triplicate. Germination tests were performed in a seed germinator (Jacobsen $\mathrm{J} 120 / \mathrm{OS}$ ) at $25^{\circ} \mathrm{C}$ for 10 days after stratification. Plants were watered with $5 \mathrm{ml}$ of deionized water during germination test. After the experiment the plant yield from each plate was dried to the constant weight, mineralized, and multielemental analysis by ICP-OES was carried out (three measurements of each probe).

\section{Analytical Methods}

Baltic Seaweeds-Zn, Residues-Zn, and Fucus-Zn (0.5 g), and germinated plant (whole mass from the plate) were purified from organic matter with concentrated nitric acid - 69\% $\mathrm{m} / \mathrm{m}(5 \mathrm{ml})$, spectrally pure (Suprapur, Merck, USA) in teflon bombs in a Milestone Start D microwave oven (USA). The select parameters of the process assured the complete digestion of samples. After mineralization, samples were diluted 10 times with re-demineralized water (Millipore Simplicity) and underwent multielemental analysis. The concentration of elements in digested biomass was determined by ICP-OES Varian-Vista MPX (Australia), equipped with an ultrasonic nebulizer CETAC U5000AT+. The analyses were carried out in a Laboratory Accredited by the Polish Centre of Accreditation (PCA) according to PNEN ISO/IEC 17025:2005. Quality assurance of the test results was achieved by using Combined Quality Control Standard from Ultra Scientific, USA. The samples were analyzed in three repeats (the reported results of analyses were arithmetic mean, the relative standard deviation was $<5 \%$ ).

\section{Statistical Analysis}

Obtained results were statistically elaborated upon using Statistica software v. 9.0. Normality of the distribution was tested with the use of Shapiro-Wilk Test. BrownForsyth test was used for the analysis of homogenity of variance for normal distribution. In the groups that fulfilled the condition of homogenity of variance, an F-test was conducted. Statistical tests for other-than-normal distribution were carried out using Kruskal-Wallis test. It was assumed that the results were statistically significant at $p<0.05$ and significant at $\mathrm{p}<0.1$. 
Table 1. The content of nutrients in biomass enriched with zinc.

\begin{tabular}{|l|c|c|c|c|c|c|c|c|c|}
\hline \multirow{2}{*}{ Material } & \multicolumn{7}{|c|}{ The content of nutrients in biomass $\left[\mathrm{mg} \cdot \mathrm{kg}^{-1}\right]$} \\
\cline { 2 - 10 } & $\mathrm{Zn}$ & $\mathrm{Fe}$ & $\mathrm{Mn}$ & $\mathrm{Cu}$ & $\mathrm{Ca}$ & $\mathrm{S}$ & $\mathrm{Mg}$ & $\mathrm{P}$ & $\mathrm{K}$ \\
\hline $\begin{array}{l}\text { Baltic } \\
\text { Seaweeds }\end{array}$ & $227 \pm 34$ & $3,203 \pm 640$ & $366 \pm 50$ & $80.7 \pm 12.1$ & $17,193 \pm 3,439$ & $12,756 \pm 2,551$ & $3,836 \pm 767$ & $810 \pm 121$ & $3,259 \pm 652$ \\
\hline $\begin{array}{l}\text { Baltic } \\
\text { Seaweeds-Zn }\end{array}$ & $30,100 \pm 6,020$ & $5,070 \pm 1,010$ & $94.1 \pm 14.1$ & $51.6 \pm 7.7$ & $11,900 \pm 2,380$ & $23,900 \pm 4,790$ & $2,130 \pm 426$ & $2,130 \pm 425$ & $1,240 \pm 242$ \\
\hline Residues & $98.0 \pm 14.7$ & $4,493 \pm 899$ & $531 \pm 80$ & $16.4 \pm 2.5$ & $14,580 \pm 2,916$ & $24,420 \pm 4,884$ & $6,788 \pm 1,358$ & $2,452 \pm 490$ & $21,082 \pm 42,164$ \\
\hline Residues-Zn & $29,700 \pm 5,940$ & $4,540 \pm 907$ & $105 \pm 16$ & $28.5 \pm 4.3$ & $5,070 \pm 1,020$ & $20,800 \pm 4,150$ & $1,130 \pm 226$ & $1,970 \pm 2,390$ & $1,370 \pm 273$ \\
\hline Fucus & $35.9 \pm 5.4$ & $311 \pm 46.7$ & $57.6 \pm 8.6$ & $2.35 \pm 0.35$ & $9,756 \pm 1,951$ & $17,540 \pm 3,508$ & $4,099 \pm 820$ & $921 \pm 138$ & $2,125 \pm 425$ \\
\hline Fucus-Zn & $59,400 \pm 11,880$ & $400 \pm 60$ & $14.7 \pm 2.2$ & $23.2 \pm 3.5$ & $8,750 \pm 1,750$ & $23,700 \pm 2,730$ & $2,020 \pm 403$ & $519 \pm 78$ & $1,350 \pm 227$ \\
\hline
\end{tabular}

\section{Results}

The element content of different types of biomass enriched with zinc(II) ions in the biosorption process is presented in Table 1. The highest biosorption capacity for zinc(II) ions was observed for Fucus sp. and it was evaluated as $6 \%$. It was twice higher than biosorption capacity of Baltic Seaweeds and Residues. Multielemental analysis showed that macroalgae enriched with zinc(II) ions constituted a source of significant amounts of elements important for plants such as iron, calcium, sulfur, and potassium. After biosorption there is a visible $\mathrm{Ca}, \mathrm{K}$, and $\mathrm{Mg}$ content decrease (except Residues), in which alkali metals were exchanged with micronutrients (biosorption due to ion exchange).

The influence of different methods of micronutrient fertilization on the mass and content of $\mathrm{Zn}, \mathrm{Cu}, \mathrm{Fe}, \mathrm{K}$, and $\mathrm{Ca}$ are presented in Table 2 (elements with normal distribution in statistical result analysis). In Table 3 content of $\mathrm{Mn}, \mathrm{Mg}$, $\mathrm{Na}, \mathrm{S}$, and $\mathrm{Si}$ (elements with other-than-normal distribution in statistical result analysis) is presented. It was shown that increasing doses of zinc led to the increase of plant mass and zinc content. For most cases statistically significant differences were found in zinc, potassium, and calcium content in plants. In Table 2 the antagonism between $\mathrm{Zn} / \mathrm{Fe}$, $\mathrm{Zn} / \mathrm{Mn}$, and $\mathrm{Zn} / \mathrm{Ca}$ is visible; with increasing zinc doses, decreases of $\mathrm{Fe}, \mathrm{MN}$, and $\mathrm{Ca}$ content in plants was obtained. Zinc was changed with these ions due to biosorption. Analysis of changes in plant mass in relation to different zinc doses enabled us to find optimal doses of zinc for the fertilization of Lepidium sativum ( $4 \mathrm{mg} \mathrm{Zn} / \mathrm{dish}$ ).

The most important correlations between elements in plants are presented in Table 4 . The positive correlation between zinc and plant mass was observed (0.55). The existence of negative correlations between zinc and other cationic elements such as $\mathrm{Ca}(-0.68), \mathrm{K}(-0.62), \mathrm{Na}(-0.61)$, $\mathrm{Mg}(-0.51)$, and $\mathrm{Mn}(-0.50)$ was found. It confirms observations about antagonism between these elements and zinc, and explains the influence of ion exchange during biosorption, which is also related to these ions. Table 5 presents the comparison of element correlations for plants fertilized with traditional fertilizer and with the use of new components with micronutrients. It can be observed that the application of biocomponents with zinc slightly increased some positive correlations between substantial elements, while negative relationships were weakened. Algae components increased the correlation between $\mathrm{Mn}$ and $\mathrm{Mg}$ and between $\mathrm{S}$ and Si. Also, correlation between $\mathrm{Fe}$ and $\mathrm{Cu}$ was improved. Reduction of antagonistic influence of zinc on $\mathrm{Ca}, \mathrm{K}, \mathrm{Na}$, and $\mathrm{S}$ also was remarkable. The content of $\mathrm{Zn}$ (II) in plants fertilized with different zinc components is shown in Fig. 1. Increased doses of $\mathrm{Zn}$ (II) result in increased content of this micronutrient in plants.

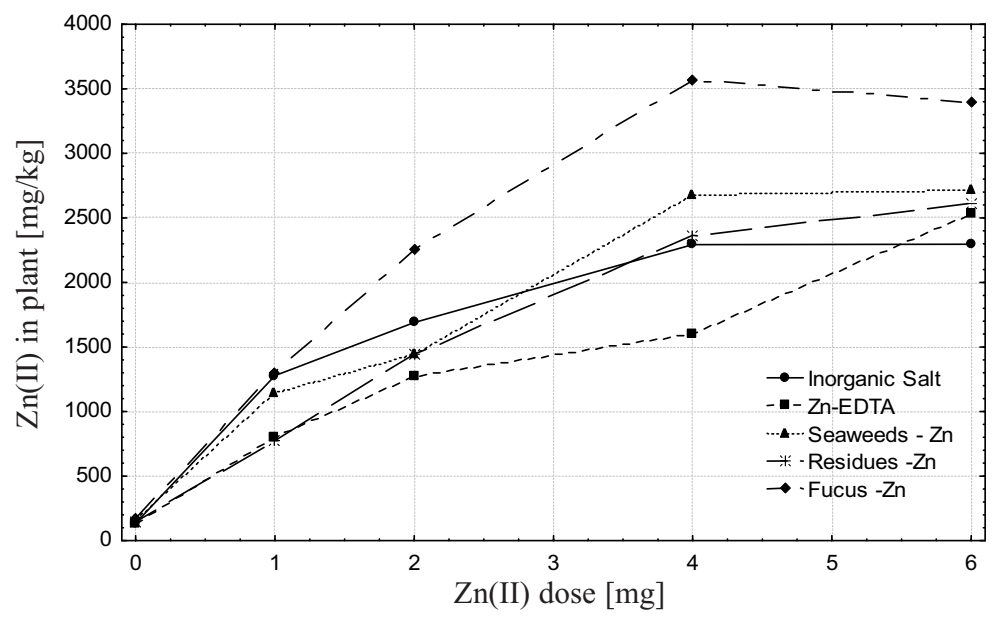

Fig. 1. The content of zinc in plants fertilized with different zinc components. 


\begin{tabular}{|c|c|c|c|c|c|c|c|c|c|c|c|c|c|c|c|c|c|c|c|c|}
\hline \multirow[b]{2}{*}{ ש } & ह & $\stackrel{\circ}{\dot{m}}$ & $\mid \frac{a}{\gtrless}$ & 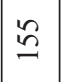 & $\stackrel{e}{\stackrel{0}{r}}$ & $\stackrel{n}{n}$ & $\tilde{n}$ & $\stackrel{\infty}{-}$ & $\stackrel{\infty}{\stackrel{\infty}{\sim}}$ & $\stackrel{\Xi}{\Xi}$ & $\stackrel{8}{=}$ & ஜे & 卢 & $\stackrel{n}{\sim}$ & $\begin{array}{l}\mathscr{D}^{\circ} \\
\stackrel{0}{ }\end{array}$ & $\overline{\vec{\sim}}$ & 总 & $\stackrel{8}{-}$ & $\underset{m}{m}$ & $\stackrel{ \pm}{\sim}$ \\
\hline & $\sum_{\bar{\Xi}}^{\Xi}$ & 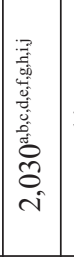 & 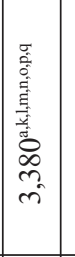 & 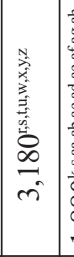 & 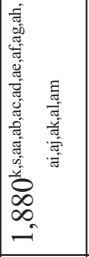 & 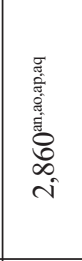 & 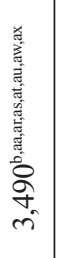 & 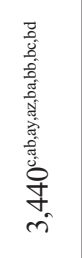 & 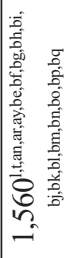 & 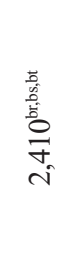 & 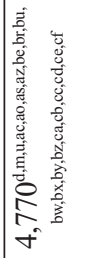 & 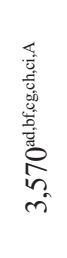 & 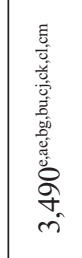 & 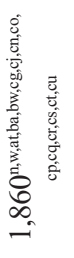 & 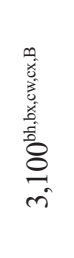 & 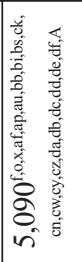 & 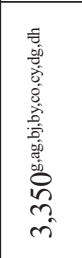 & 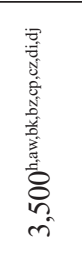 & 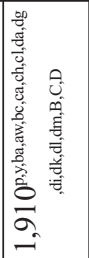 & 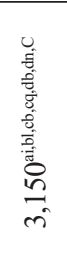 \\
\hline \multirow[b]{2}{*}{ 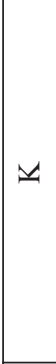 } & क & $\approx$ & 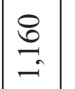 & $\stackrel{+}{\stackrel{4}{4}}$ & 范 & 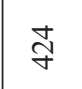 & $\begin{array}{l}\infty \\
\infty \\
\infty \\
-1\end{array}$ & $\stackrel{8}{\circ}$ & $\underset{i}{\stackrel{i}{0}}$ & $\stackrel{\overbrace{}}{\beth}$ & 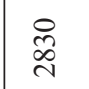 & \&े & 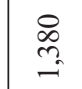 & $\stackrel{0}{\cong}$ & $\underset{\substack{i \\
i}}{ }$ & 荇 & $\stackrel{\infty}{\Omega}$ & $\stackrel{\circ}{n}$ & $\vec{n}$ & $\hat{n}$ \\
\hline & 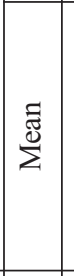 & 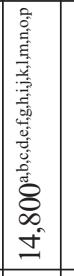 & 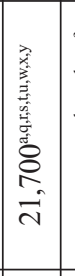 & 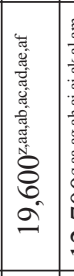 & 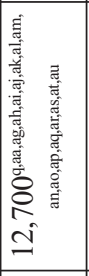 & 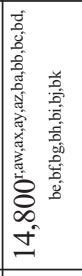 & 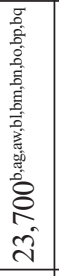 & 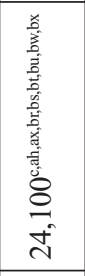 & 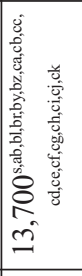 & 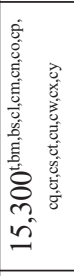 & 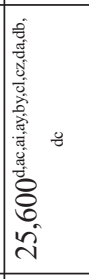 & 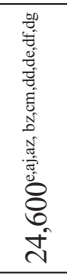 & 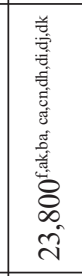 & 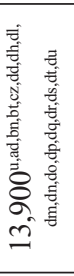 & 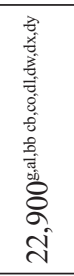 & 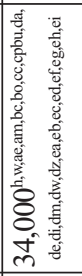 & 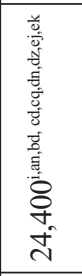 & 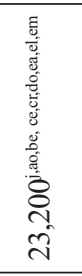 & 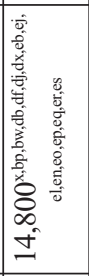 & 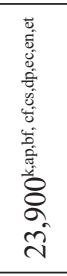 \\
\hline \multirow[b]{2}{*}{ 语 } & की & $\stackrel{m}{2}$ & $\left|\begin{array}{l}0 \\
0 \\
0 \\
0\end{array}\right|$ & $\frac{n}{6}$ & $\begin{array}{l}\text { \& } \\
\infty\end{array}$ & $\stackrel{\infty}{\stackrel{\infty}{-}}$ & 品 & $\stackrel{\infty}{\check{c}}$ & $\stackrel{n}{=}$ & $\hat{\tilde{a}}$ & ల్ల & $\stackrel{m}{=}$ & $\begin{array}{l}\infty \\
\stackrel{\infty}{\infty}\end{array}$ & $\ddot{q}$ & $\stackrel{+}{\stackrel{f}{\oplus}}$ & $\bar{\sim}$ & $\hat{\mathrm{m}}$ & $\ddot{\ddot{g}}$ & $\stackrel{\text { }}{=}$ & $\stackrel{\simeq}{=}$ \\
\hline & $\sum^{\text {चี }}$ & $\stackrel{\stackrel{\rho}{\partial}}{\Xi}$ & 总 & $\stackrel{\square}{=}$ & 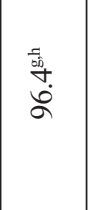 & $\stackrel{\mathbb{Z}}{\mathcal{O}}$ & 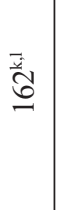 & 耐 & 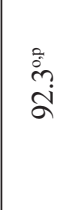 & 蒿 & 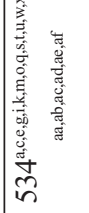 & 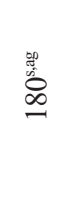 & $\stackrel{\stackrel{\bar{d}}{\omega}}{\stackrel{\infty}{a}}$ & 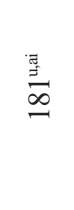 & $\frac{\sqrt{i}}{\stackrel{1}{*}}$ & 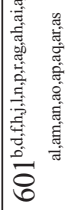 & 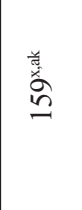 & $\begin{array}{l}\frac{\sqrt{3}}{\partial} \\
\frac{0}{0}\end{array}$ & 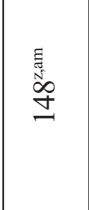 & 跑 \\
\hline \multirow[b]{2}{*}{ U } & की & 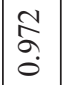 & הุ & $\stackrel{\infty}{\infty}$ & 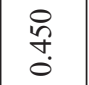 & $\stackrel{m}{i}$ & $\vec{n}$ & $\underset{\text { in }}{\stackrel{ \pm}{n}}$ & $\begin{array}{l}8 \\
\infty \\
\infty \\
0 \\
0\end{array}$ & $\stackrel{\hat{\sigma}}{r}$ & \begin{tabular}{l}
\multirow{1}{*}{} \\
$\infty$ \\
$\infty$ \\
0
\end{tabular} & 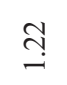 & $\stackrel{\bar{i}}{i}$ & $\stackrel{\substack{+\stackrel{+}{*}}}{ }$ & $\stackrel{n}{\sigma}$ & $\begin{array}{l}\tilde{\alpha} \\
\stackrel{2}{0}\end{array}$ & $\begin{array}{l}\stackrel{0}{0} \\
\stackrel{\infty}{0}\end{array}$ & $\stackrel{\leftrightarrow}{a}$ & $\stackrel{\tilde{n}}{\stackrel{n}{-}}$ & $\stackrel{8}{8}$ \\
\hline & $\sum_{\Sigma}^{\Xi}$ & $\mid$ & $\mid \begin{array}{l}0 \\
\hat{1} \\
n \\
n \\
2\end{array}$ & $\stackrel{\stackrel{\Xi}{\circ}}{=}$ & 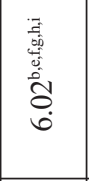 & $\stackrel{\infty}{=}$ & 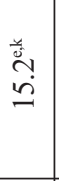 & 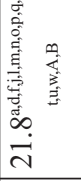 & 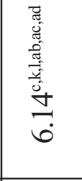 & $\stackrel{m}{n}$ & 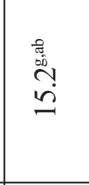 & $\stackrel{\mathbb{E}}{=}$ & $\underset{\mathrm{I}}{\mathrm{I}}$ & $\stackrel{\text { İ }}{\text { I }}$ & $\begin{array}{l}\infty \\
\stackrel{\bigcup}{I}\end{array}$ & $\stackrel{8}{\ddot{g}}$ & $\stackrel{\check{I}}{\mathrm{I}}$ & 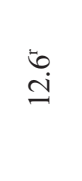 & $\stackrel{i}{i}$ & 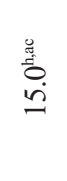 \\
\hline \multirow[b]{2}{*}{ సี } & की & $\grave{\grave{I}}$ & $\frac{7}{m}$ & 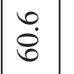 & $\stackrel{\infty}{\sim}$ & : & ¿̊. & \begin{tabular}{l}
$b^{\circ}$ \\
\hdashline
\end{tabular} & बे & $\stackrel{\partial}{\exists}$ & in & $\Xi$ & $\stackrel{\infty}{\sim}$ & 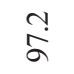 & 赵 & $\stackrel{n}{=}$ & 茡 & $\vec{J}$ & $\widetilde{ల}$ & : \\
\hline & 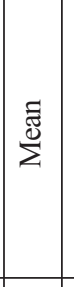 & 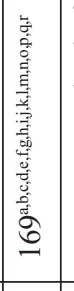 & 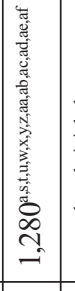 & 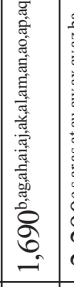 & 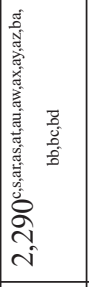 & 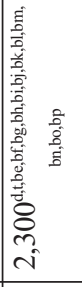 & 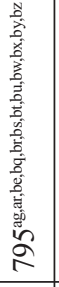 & 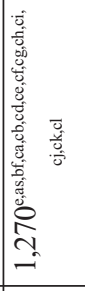 & 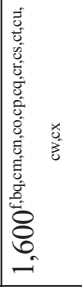 & 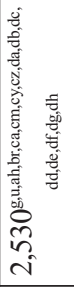 & 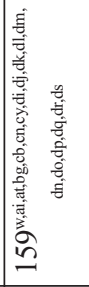 & 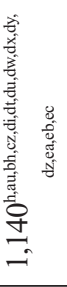 & 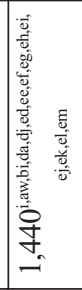 & 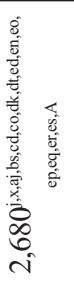 & 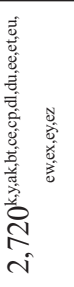 & 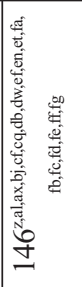 & 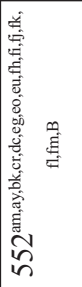 & 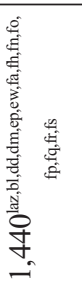 & 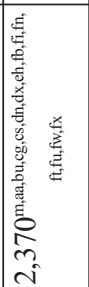 & 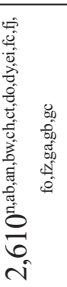 \\
\hline \multirow{2}{*}{ 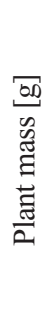 } & के & 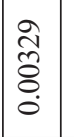 & $\begin{array}{l}8 \\
\stackrel{8}{0} \\
\stackrel{8}{0} \\
0\end{array}$ & $\begin{array}{l}0 \\
\infty \\
0 \\
0 \\
0 \\
0\end{array}$ & 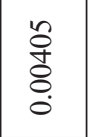 & 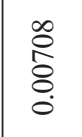 & 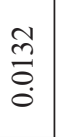 & 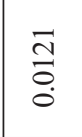 & $\begin{array}{l}\tilde{n} \\
\tilde{o} \\
\vdots \\
0 \\
0\end{array}$ & $\begin{array}{l}\overrightarrow{0} \\
\stackrel{0}{0} \\
0\end{array}$ & 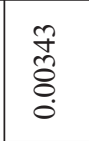 & $\begin{array}{l}\hat{n} \\
\stackrel{0}{0} \\
\vdots \\
0\end{array}$ & $\begin{array}{l}\text { oे } \\
\text { ஜิ } \\
\text {. }\end{array}$ & $\frac{n}{n}$ & 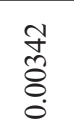 & $\begin{array}{l}\vec{n} \\
\tilde{\delta} \\
0 \\
0 \\
0\end{array}$ & $\begin{array}{l}\frac{a}{\infty} \\
\text { oे } \\
0 \\
0 \\
0\end{array}$ & $\begin{array}{l}\text { ôे } \\
\text { ठิ. } \\
0\end{array}$ & $\frac{n}{a}$ & 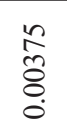 \\
\hline & $\sum_{\bar{E}}^{\Xi}$ & $\mid \begin{array}{l}0 \\
\vdots \\
0 \\
0\end{array}$ & $\mid \begin{array}{l}\infty \\
0 \\
0 \\
0 \\
0\end{array}$ & 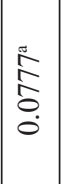 & 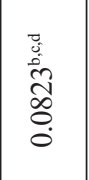 & $\begin{array}{l}\text { ô } \\
\stackrel{0}{0} \\
0\end{array}$ & 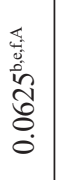 & 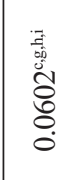 & $\begin{array}{l}\hat{o} \\
0 \\
0 \\
0\end{array}$ & 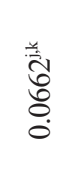 & $\begin{array}{l}\stackrel{\circ}{0} \\
\stackrel{0}{0}\end{array}$ & $\begin{array}{l}\mathscr{0} \\
\stackrel{0}{0} \\
\stackrel{0}{0}\end{array}$ & 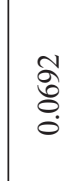 & 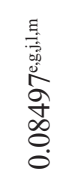 & $\begin{array}{l}\stackrel{0}{0} \\
\stackrel{2}{\hat{0}} \\
\stackrel{0}{0}\end{array}$ & $\begin{array}{l}\stackrel{i}{n} \\
\hat{0} \\
0 \\
0\end{array}$ & 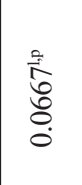 & $\begin{array}{l}\text { ôे } \\
\text { o. }\end{array}$ & 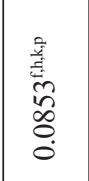 & $\begin{array}{l}\stackrel{5}{+5} \\
\stackrel{+}{0} \\
\stackrel{0}{0}\end{array}$ \\
\hline \multicolumn{2}{|c|}{ 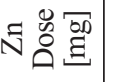 } & ' & - & $\sim$ & $\nabla$ & 0 & - & $N$ & 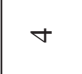 & 0 & 0 & - & $\sim$ & $\nabla$ & 0 & 0 & - & $\sim$ & $\nabla$ & 0 \\
\hline \multicolumn{2}{|c|}{$\begin{array}{l}0 \\
0 \\
0\end{array}$} & 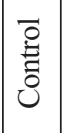 & \multicolumn{4}{|c|}{ 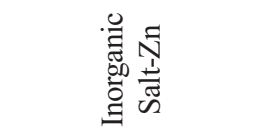 } & \multicolumn{4}{|c|}{ 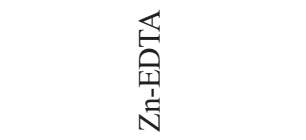 } & \multicolumn{5}{|c|}{ 量 } & \multicolumn{5}{|c|}{ 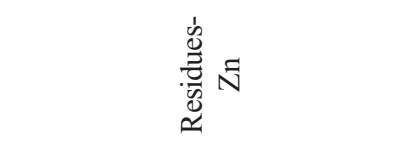 } \\
\hline
\end{tabular}




\begin{tabular}{|c|c|c|c|c|c|c|c|}
\hline \multirow[b]{2}{*}{ ש } & के & 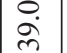 & $\stackrel{尺}{\Sigma}$ & $\frac{\infty}{m}$ & ๙ิ & $\underset{\infty}{\infty}$ & $\frac{1}{6}$ \\
\hline & $\sum_{\Sigma}^{\mathbb{E}}$ & 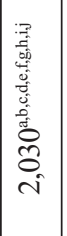 & 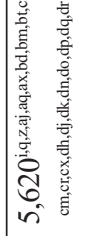 & 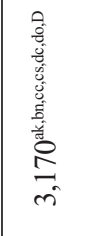 & 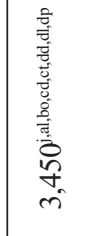 & 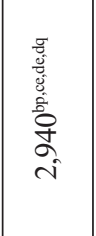 & 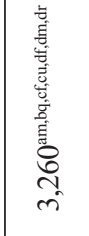 \\
\hline \multirow[b]{2}{*}{ 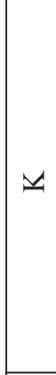 } & के & $\begin{array}{l}n \\
\infty \\
\infty\end{array}$ & 亲 & $\stackrel{8}{\circledR}$ & $\bar{n}$ & $\underset{\rightarrow}{\mathscr{D}}$ & $\stackrel{\circ}{\circ}$ \\
\hline & 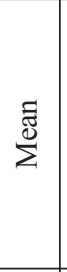 & 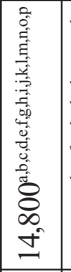 & 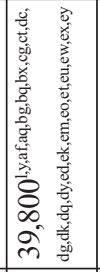 & 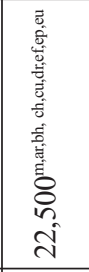 & 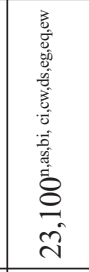 & 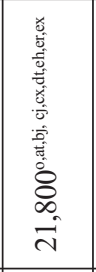 & 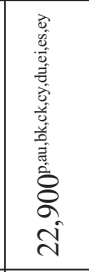 \\
\hline \multirow[b]{2}{*}{ II } & हे & $\stackrel{3}{2}$ & $\ddot{\tilde{\gamma}}$ & $\overline{0}$ & $\stackrel{\infty}{\stackrel{\infty}{\longrightarrow}}$ & ì & $\stackrel{\circ}{\circ}$ \\
\hline & $\sum_{\Sigma}^{\tilde{E}}$ & 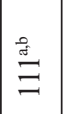 & 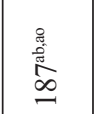 & 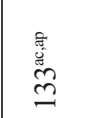 & $\begin{array}{l}\text { 嘭 } \\
\text { ले }\end{array}$ & 㺃 & 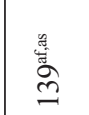 \\
\hline \multirow{2}{*}{$\exists$} & ह & 等 & సे & $\stackrel{\infty}{\stackrel{\infty}{2}}$ & $\stackrel{\text { ֻ̊ }}{-}$ & $\stackrel{\overbrace{}}{-}$ & $\overrightarrow{\vec{i}}$ \\
\hline & $\sum_{\Sigma}^{\tilde{E}}$ & $\stackrel{\substack{* \\
\sim}}{\sim}$ & $\begin{array}{l}\text { 总 } \\
\dot{b} \\
\dot{n}\end{array}$ & $\begin{array}{l}\stackrel{5}{0} \\
a\end{array}$ & పे & 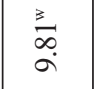 & 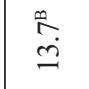 \\
\hline \multirow[b]{2}{*}{ ㄱ } & हि & $\widehat{\grave{j}}$ & $\stackrel{n}{\stackrel{n}{=}}$ & $\hat{q}$ & ฮิ & $\stackrel{\text { a }}{ }$ & 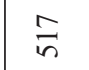 \\
\hline & 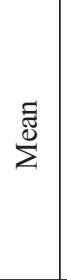 & 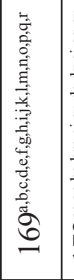 & 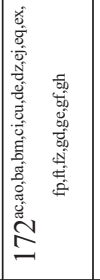 & 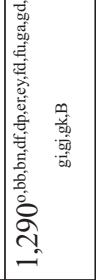 & 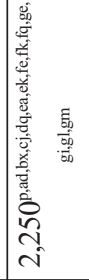 & 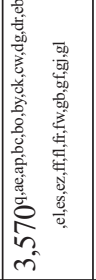 & 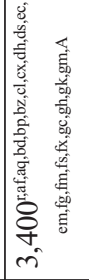 \\
\hline \multirow{2}{*}{ 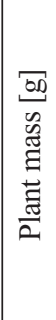 } & हे & 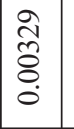 & $\begin{array}{l}\hat{n} \\
\stackrel{0}{0} \\
0\end{array}$ & 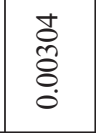 & 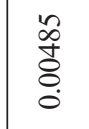 & $\begin{array}{l}\vec{\nabla} \\
\overrightarrow{0} \\
\dot{0}\end{array}$ & $\begin{array}{l}\overrightarrow{0} \\
\stackrel{\widehat{\sigma}}{0} \\
\stackrel{0}{0}\end{array}$ \\
\hline & $\sum^{\Xi ี \Xi}$ & \begin{tabular}{|l|} 
\\
\\
0 \\
0 \\
0 \\
\end{tabular} & 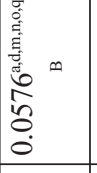 & $\begin{array}{l}\text { m} \\
\hat{0} \\
0\end{array}$ & 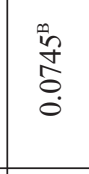 & $\begin{array}{l}\frac{c}{3} \\
\hat{a} \\
\hat{o} \\
0 \\
0\end{array}$ & $\begin{array}{l}\text { 苂 } \\
\stackrel{0}{0} \\
0 \\
0\end{array}$ \\
\hline \multicolumn{2}{|c|}{ 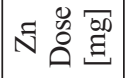 } & ' & 0 & - & $\sim$ & $\nabla$ & 0 \\
\hline \multicolumn{2}{|c|}{$\begin{array}{l}0 \\
\text { oे } \\
0\end{array}$} & 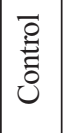 & \multicolumn{5}{|c|}{ 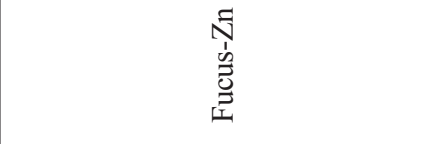 } \\
\hline
\end{tabular}

The best effect was achieved for Fucus-Zn while the weakest biofortification was obtained for Zn-EDTA.

As was presented in Fig. 2, Transfer Factor of $\mathrm{Zn}$ (II) was inversely correlated with $\mathrm{Zn}$ (II) dose. This means that the assimilation of micronutrients by plants decreases with increasing doses. The dependence between zinc dose and mass of plant is presented in Fig. 3. The highest values of plant mass were obtained for dose $4 \mathrm{mg} \mathrm{Zn/dish.} \mathrm{Further}$ increasing of zinc dose led to a decrease of plant mass. Taking into account the impact of different zinc doses on the mass of plant and micronutrient content, it can be deduced that $4 \mathrm{mg} \mathrm{Zn} \mathrm{per} \mathrm{dish} \mathrm{is} \mathrm{the} \mathrm{best} \mathrm{dose} \mathrm{for} \mathrm{micronu-}$ trient biofortification of Lepidium sativum.

\section{Discussion}

In the present study, it was observed that zinc fertilization with the use of different fertilizer biocomponents led to biofortification of plants. Six experimental groups (Baltic Seaweed, Residues, Fucus and the control groups: Znchelate, inorganic salt, and untreated) were tested in triplicate as fertilizer materials. The biomass from each plate was collected separately, and multielemental composition was analyzed (also in triplicate; $\mathrm{N}=54$ ).

The biosorption of the three different types of algae biomass led to the enrichment of biological material with zinc. The biomass of Fucus sp. was shown to be the most efficient biosorbent. $\mathrm{Zn}(\mathrm{II})$ content in Baltic Seaweed and Residues was two times lower $(30,100$ and $29,700 \mathrm{mg} / \mathrm{kg}$ respectively). The comparable biosorption capacity of Baltic Seaweed and Residues suggests that extraction procedures did not significantly affect the sorption capacity of biological material. It also confirms that the surface of the biomass (cell wall) mainly participates in the biosorption process. All tested materials were shown to be a rich source of other micro- and macronutrients, especially $\mathrm{Fe}, \mathrm{Ca}, \mathrm{S}$, $\mathrm{Mg}$, and $\mathrm{K}$ (Table 1). It was proved that increasing zinc doses led to increased plant mass and zinc content in plants (Table 2, Fig. 1). The differences in plant yield between groups in the experiment were also observed (Table 2). The mass of plants fertilized with zinc was higher than in the control group $(0.0706 \mathrm{~g})$ in all groups fertilized with 4 and $6 \mathrm{mg}$ per dish besides Zn-EDTA and Inorganic salt at 6 $\mathrm{mg}$ dose $(0.0662 \mathrm{~g}$ and $0.0703 \mathrm{~g})$. The conducted experiments showed that zinc fertilization with the use of Fucus$\mathrm{Zn}$ was the most efficient, while Zn-EDTA was characterized by the lowest transfer of $\mathrm{Zn}$ (II) ions. Fig. 2 shows that the transfer factor of $\mathrm{Zn}$ (II) decreased with increasing zinc doses. It showed that overfertilization leads to lower assimilation of nutrients by plants.

Conducted experiments showed that there is a positive correlation between plant mass (0.55) and $\mathrm{Zn}$ dose (Table 4). Zinc is one of the most important micronutrients and plays an important role as catalyst, structural, and regulatory ion, being necessary for various biochemical reactions and physiological functions such as photosynthesis, respiration, and chlorophyll biosynthesis [23], and as it was shown has influence on plant mass. The strong correlation was inde- 
Table 3. Content of nutrients in plants $\left(\mathrm{mg} \cdot \mathrm{kg}^{-1}\right)$. Elements with other than normal distribution in statistical result analysis.

\begin{tabular}{|c|c|c|c|c|c|c|c|c|c|c|c|}
\hline \multirow{2}{*}{ Probe } & \multirow{2}{*}{$\begin{array}{c}\mathrm{Zn} \text { Dose } \\
{[\mathrm{mg}]}\end{array}$} & \multicolumn{2}{|c|}{ Mn } & \multicolumn{2}{|c|}{$\mathrm{Mg}$} & \multicolumn{2}{|c|}{$\mathrm{Na}$} & \multicolumn{2}{|c|}{ S } & \multicolumn{2}{|c|}{$\mathrm{Si}$} \\
\hline & & Mean & $\mathrm{SD}$ & Mean & $\mathrm{SD}$ & Mean & $\mathrm{SD}$ & Mean & $\mathrm{SD}$ & Mean & SD \\
\hline Control & - & 29.1 & 0.0411 & 3,760 & 127 & 5,630 & 936 & 11,300 & 1,090 & 169 & 12.9 \\
\hline \multirow{4}{*}{$\begin{array}{c}\text { Inorganic } \\
\text { Salt-Zn }\end{array}$} & 1 & 35.9 & 1.11 & 5,960 & 77.8 & 7,290 & 194 & 22,100 & 192 & 1,280 & 312 \\
\hline & 2 & 33.5 & 1.57 & 5,770 & 143 & 6,720 & 1,210 & 21,000 & 608 & 1,690 & 60.6 \\
\hline & 4 & $28.1^{\mathrm{a}, \mathrm{A}}$ & 1.43 & 3,800 & 92.4 & $2,680^{\mathrm{a}, \mathrm{b}}$ & 303 & 12,300 & 729 & $2,290^{\mathrm{a}, \mathrm{b}}$ & 268 \\
\hline & 6 & 34.0 & 1.51 & 5,790 & 296 & 4,540 & 575 & 19,700 & 698 & 2,300 & 6.00 \\
\hline \multirow{4}{*}{ Zn-EDTA } & 1 & 34.6 & 0.395 & 5,690 & 190 & 7,390 & 1,280 & 23,200 & 734 & 795 & 69.4 \\
\hline & 2 & 35.9 & 2.84 & 5,330 & 289 & 7,280 & 1,960 & 22,500 & 1,220 & 1,270 & 78.6 \\
\hline & 4 & $24.6^{\mathrm{b}, \mathrm{c}, \mathrm{B}}$ & 4.22 & $3,190^{\mathrm{A}, \mathrm{B}}$ & 615 & $2,710^{\mathrm{c}, \mathrm{A}}$ & 687 & 12,600 & 2,550 & $1,600^{\mathrm{c}, \mathrm{d}, \mathrm{A}}$ & 299 \\
\hline & 6 & 31.9 & 1.95 & 4,760 & 85.0 & 4,520 & 896 & 20,100 & 497 & 2,530 & 119 \\
\hline \multirow{5}{*}{$\begin{array}{c}\text { Baltic } \\
\text { Seaweeds } \\
-\mathrm{Zn}\end{array}$} & 0 & $72.4^{\mathrm{b}, \mathrm{A}}$ & 15.6 & 6,040 & 417 & 10,700 & 1,340 & 19,400 & 1,650 & $159^{\mathrm{a}, \mathrm{c}}$ & 15.0 \\
\hline & 1 & 43.3 & 0.0252 & 6,410 & 113 & 10,000 & 972 & 22,320 & 838 & 1,140 & 117 \\
\hline & 2 & 42.7 & 1.49 & 6,640 & 291 & 8,220 & 940 & 22,300 & 654 & 1,440 & 248 \\
\hline & 4 & 30.1 & 2.23 & 3,590 & 247 & 4,730 & 1,560 & 11,700 & 722 & 2,680 & 97.2 \\
\hline & 6 & 44.1 & 0.574 & 6,330 & 164 & 8,700 & 1,300 & 23,100 & 952 & 2,720 & 368 \\
\hline \multirow{5}{*}{$\begin{array}{l}\text { Residues- } \\
\text { Zn }\end{array}$} & 0 & $125^{\mathrm{a}, \mathrm{b}}$ & 24.3 & 6,370 & 88.6 & $13,100^{\mathrm{a}, \mathrm{A}}$ & 1,500 & 21,000 & 1,020 & $146^{\mathrm{b}, \mathrm{d}}$ & 11.5 \\
\hline & 1 & 43.9 & 0.463 & $6,710^{\mathrm{A}}$ & 191 & 8,770 & 220 & 23,100 & 937 & 552 & 404 \\
\hline & 2 & 42.4 & 2.63 & 6,650 & 313 & 8,180 & 206 & 22,000 & 457 & 1,440 & 241 \\
\hline & 4 & 31.0 & 0.321 & $3,530^{\mathrm{C}}$ & 209 & 3,850 & 246 & 11,700 & 108 & 2,370 & 323 \\
\hline & 6 & 43.6 & 0.945 & 6,280 & 206 & 7,650 & 1,080 & 22,800 & 328 & 2,610 & 266 \\
\hline \multirow{5}{*}{ Fucus-Zn } & 0 & $52.9^{\mathrm{B}}$ & 8.17 & $7,210^{\mathrm{B}, \mathrm{C}}$ & 661 & $19,400^{\mathrm{b}, \mathrm{c}}$ & 2,180 & 23,200 & 3,090 & $172^{\mathrm{A}}$ & 17.5 \\
\hline & 1 & 36.4 & 1.48 & 5,790 & 229 & 8,670 & 414 & 20,400 & 256 & 1,290 & 407 \\
\hline & 2 & 34.8 & 1.02 & 5,580 & 198 & 8,500 & 626 & 20,200 & 397 & 2,250 & 102 \\
\hline & 4 & 31.8 & 1.46 & 5,200 & 385 & 7,620 & 497 & 19,700 & 864 & 3,570 & 109 \\
\hline & 6 & 35.4 & 2.51 & 5,710 & 162 & 8,310 & 1,000 & 21,400 & 599 & 3,400 & 517 \\
\hline
\end{tabular}

Statistically significant differences for a given nutrients without normal distribution a,b... $(p<0.05),{ }^{A, B \ldots}(\mathrm{p}<0.1), \mathrm{N}=54$.

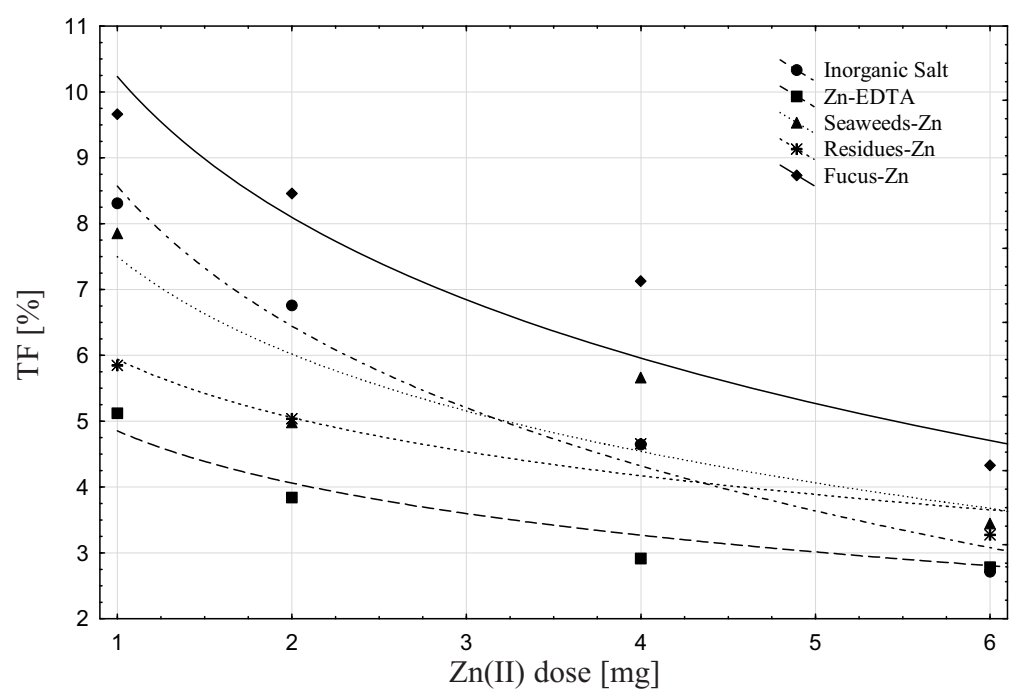

Fig. 2. The change in zinc transfer factor for different zinc doses and different components. 
Table 4. Correlation matrix of element content in plants $(\mathrm{N}=54, \mathrm{p}<0.05)$.

\begin{tabular}{|c|c|c|c|c|c|c|c|c|c|c|c|c|c|}
\hline & $\begin{array}{l}\text { Plant } \\
\text { mass }\end{array}$ & $\mathrm{Al}$ & $\mathrm{Ca}$ & $\mathrm{Cr}$ & $\mathrm{Cu}$ & $\mathrm{Fe}$ & $\mathrm{K}$ & $\mathrm{Mg}$ & $\mathrm{Mn}$ & $\mathrm{Na}$ & $\mathrm{P}$ & S & $\mathrm{Si}$ \\
\hline $\mathrm{Al}$ & 0.084 & & & & & & & & & & & & \\
\hline $\mathrm{Ca}$ & -0.47 & 0.52 & & & & & & & & & & & \\
\hline $\mathrm{Cr}$ & -0.26 & 0.076 & 0.27 & & & & & & & & & & \\
\hline $\mathrm{Cu}$ & -0.46 & 0.25 & 0.28 & 0.059 & & & & & & & & & \\
\hline $\mathrm{Fe}$ & -0.03 & 0.86 & 0.61 & 0.13 & 0.12 & & & & & & & & \\
\hline $\mathrm{K}$ & -0.43 & 0.33 & 0.88 & 0.34 & 0.23 & 0.40 & & & & & & & \\
\hline $\mathrm{Mg}$ & -0.33 & 0.17 & 0.62 & 0.27 & 0.06 & 0.26 & 0.68 & & & & & & \\
\hline $\mathrm{Mn}$ & -0.076 & 0.76 & 0.67 & 0.31 & 0.10 & 0.87 & 0.57 & 0.40 & & & & & \\
\hline $\mathrm{Na}$ & -0.42 & 0.35 & 0.85 & 0.35 & 0.14 & 0.38 & 0.94 & 0.65 & 0.53 & & & & \\
\hline $\mathrm{P}$ & -0.40 & -0.23 & 0.11 & 0.22 & 0.18 & -0.13 & 0.19 & 0.60 & -0.12 & 0.18 & & & \\
\hline $\mathrm{S}$ & -0.43 & -0.064 & 0.27 & 0.23 & 0.33 & 0.00 & 0.43 & 0.57 & 0.09 & 0.35 & 0.62 & & \\
\hline $\mathrm{Si}$ & -0.21 & 0.82 & 0.73 & 0.25 & 0.22 & 0.94 & 0.56 & 0.38 & 0.93 & 0.53 & -0.053 & 0.11 & \\
\hline $\mathrm{Zn}$ & 0.55 & -0.25 & -0.68 & -0.21 & -0.21 & -0.37 & -0.62 & -0.51 & -0.50 & -0.61 & -0.14 & -0.31 & -0.49 \\
\hline
\end{tabular}

Strong correlations were bolded.

pendent of zinc fertilization (it was observed for traditional zinc fertilizers and new biocomponents). It can be explained by the participation of zinc in the synthesis of plant growth hormones [24].

The statistical analysis of multielement content of plants showed the presence of some element interdependencies. Zinc was negatively correlated with $\mathrm{Ca}(-0.68)$, $\mathrm{K}(-0.62), \mathrm{Na}(-0.61), \mathrm{Mg}(-0.51)$, and $\mathrm{Mn}(-0.50)$ (Table 4). It can be explained by the divalent character of these cations and their mutual competition as a result of this feature or removal of these ions during the biosorption process.
Alkali metal ions were exchanged with zinc ions during biodorption (Table 1). In Table 2 antagonism between $\mathrm{Zn} / \mathrm{Fe}, \mathrm{Zn} / \mathrm{Mn}, \mathrm{Zn} / \mathrm{Ca}$ is also visible - with increased zinc doses came decreases in $\mathrm{Fe}, \mathrm{Mn}$, and $\mathrm{Ca}$.

Very strong positive relationships were found between: $\mathrm{K} / \mathrm{Na}, \mathrm{Fe} / \mathrm{Si}(0.94)>\mathrm{Mn} / \mathrm{Si}(0.93)>\mathrm{Ca} / \mathrm{K}(0.88)>\mathrm{Fe} / \mathrm{Mn}$ $(0.87)>\mathrm{Al} / \mathrm{Fe}(0.86)>\mathrm{Ca} / \mathrm{Na}(0.85)>\mathrm{Si} / \mathrm{Al}(0.82)>\mathrm{Mn} / \mathrm{Al}$ $(0.76)>\mathrm{Si} / \mathrm{Ca}(0.73)$. The roles of $\mathrm{K}, \mathrm{Mg}$, and $\mathrm{Ca}$ in plant metabolism and interdependencies between these macronutrients were also described in the literature [25]. The strong correlation between trivalent ions such as $\mathrm{Al}^{3+}$ and $\mathrm{Fe}^{3+}$

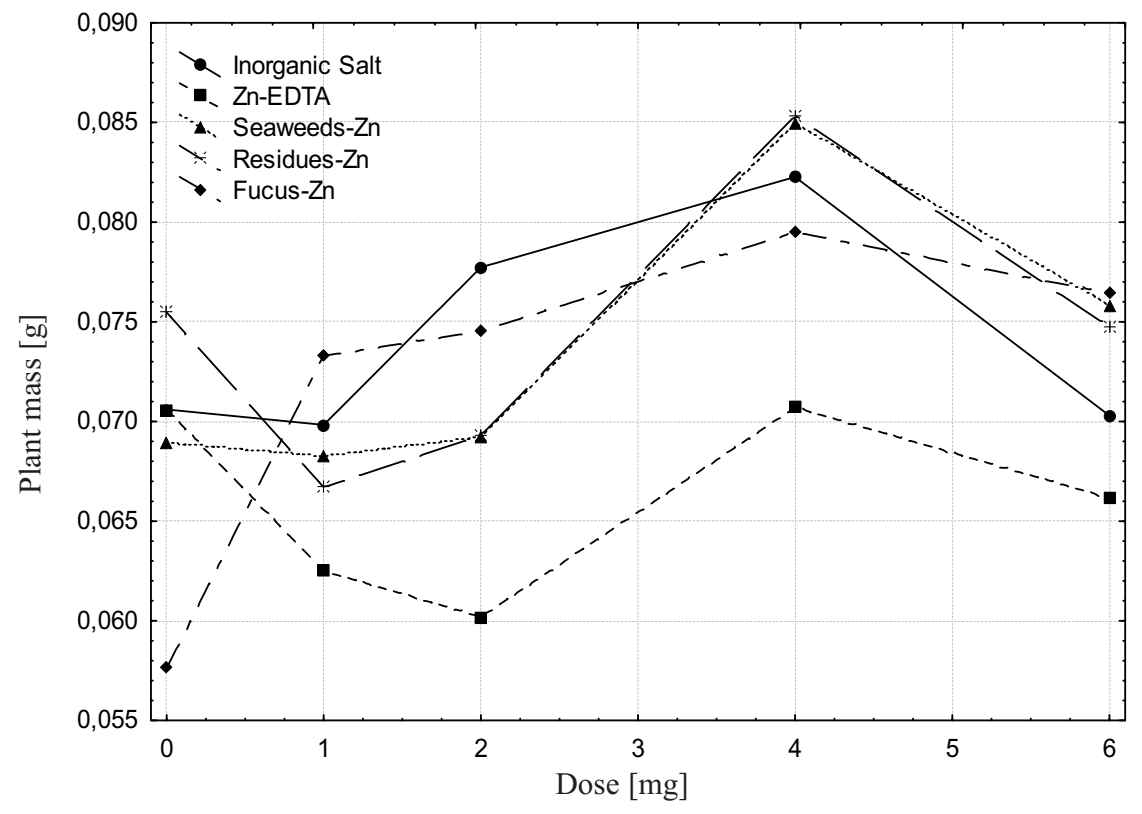

Fig. 3. The differences in mass of plants with different zinc doses and different components. 
Table 5. Comparison of element correlations for plants fertilized with traditional fertilizers and new components with micronutrients.

\begin{tabular}{|c|c|c|}
\hline & Inorganic Salt/Chelate & Algae components \\
\hline $\mathrm{Fe}: \mathrm{Cu}$ & 0.43 & 0.46 \\
\hline $\mathrm{Mn}: \mathrm{Cu}$ & 0.50 & 0.37 \\
\hline $\mathrm{Mn}: \mathrm{Mg}$ & 0.48 & 0.79 \\
\hline $\mathrm{S}: \mathrm{Cu}$ & 0.43 & 0.37 \\
\hline $\mathrm{S}: \mathrm{Fe}$ & 0.45 & 0.41 \\
\hline $\mathrm{S}: \mathrm{K}$ & 0.82 & 0.48 \\
\hline $\mathrm{Mn}: \mathrm{S}$ & 0.62 & 0.80 \\
\hline $\mathrm{Si}: \mathrm{Cu}$ & 0.61 & 0.59 \\
\hline $\mathrm{Si}: \mathrm{Fe}$ & 0.74 & 0.74 \\
\hline $\mathrm{Mn}: \mathrm{Si}$ & 0.48 & 0.82 \\
\hline $\mathrm{Zn}: \mathrm{Ca}$ & -0.82 & -0.39 \\
\hline $\mathrm{Zn}: \mathrm{K}$ & -0.83 & -0.43 \\
\hline $\mathrm{Zn}: \mathrm{Na}$ & -0.79 & -0.55 \\
\hline $\mathrm{Zn}: \mathrm{S}$ & -0.78 & -0.34 \\
\hline $\mathrm{Zn}: \mathrm{MP}$ & 0.62 & 0.65 \\
\hline
\end{tabular}

$\mathrm{MP}$ - mass of plant

$(\mathrm{r}=0.86)$ were found in our study. This correlation can constitute a result of similar radius of the hydrated ions. Strong correlations also were indicated for $\mathrm{K}: \mathrm{Mg}(0.68)>\mathrm{Mn} / \mathrm{Ca}$ $(0.67)>\mathrm{Mg} / \mathrm{Na}(0.65), \mathrm{Mg} / \mathrm{Ca}, \mathrm{P} / \mathrm{S}(0.62)>0.61 \mathrm{Fe} / \mathrm{Ca}>$ $\mathrm{Mg} / \mathrm{P}(0.60)>\mathrm{Mn} / \mathrm{K}, \mathrm{Mg} / \mathrm{S}(0.57)>\mathrm{K} / \mathrm{Si}(0.56)>\mathrm{Mn} / \mathrm{Na}$, $\mathrm{Na} / \mathrm{Si}(0.53)>\mathrm{Al} / \mathrm{Ca}(0.52)$. A synergistic trend was established for typical antagonistic pairs such as $\mathrm{Al} / \mathrm{Ca}$ and $\mathrm{Mn} / \mathrm{Ca}(\mathrm{r}=0.52$ and $\mathrm{r}=0.67$, respectively), while literature describes negative relationships between these elements [25].

\section{Conclusions}

Conducted experiments showed that the biomass of seaweeds can be enriched in the biosorption process with zinc ions with good efficiency of the process. Moreover, micronutrient ions bound to the surface of the biomass stayed in bioavailable form. The application of new preparations for micronutrient fertilization of Lepidium sativum showed that new micronutrient biopreparations can be used for the biofortification of plants and constitute an alternative tool for commercial micronutrient fertilizers.

\section{Acknowledgements}

This work was financed within the framework of a grant titled "Innovative technology of seaweed extracts components of fertilizers, feed and cosmetics" attributed to the National Centre for Research and Development in Poland, project No. PBS/1/A1/2/2012, and within the framework of a grant called "Biosorption of metal ions to the biomass of seeds of berries" attributed to the National Science Center Poland, project No. 2012/05/E/ST8/03055.

\section{References}

1. AMRUTHA V. K., KOWSALYA S., KOTHANDAPANI S. Micronutrient malnutrition profile of infants in South India. J. Hum. Nutr. Diet. 27, 54, 2014.

2. BIESALSKI H., FEUERSTEIN C., LAMBERT C., FINCO F. Fast and efficient estimation of micronutrient intake in Brazil (804.16). FASEB J. 28, 804, 2014.

3. MURGIA I., DE GARA L., GRUSAK M. A. Biofortification: how can we exploit plant science and biotechnology to reduce micronutrient deficiencies? Front. Plant Sci. 4, 2013.

4. IMPA S. M., JOHNSON-BEEBOUT S. E. Mitigating zinc deficiency and achieving high grain $\mathrm{Zn}$ in rice through integration of soil chemistry and plant physiology research. Plant Soil 361, 3, 2012.

5. HUMAYAN KABIR A., SWARAZ A. M., STANGOULIS J. Zinc-deficiency resistance and biofortification in plants. J. Plant Nutr. Soil Sci. 177, 311, 2014.

6. KONKOL N. R., MCNAMARA C. J., BEARCE-LEE K., KUNOH, H., MITCHELL R. Novel method of micronutrient application increases radish (Raphanus sativus) and shirona (Brassica Rapa var. Pekinensis) biomass. J. Plant Nutr. 35, 471, 2012.

7. RAWAT N., NEELAM K., TIWARI V. K., DHALIWAL H. S. Biofortification of cereals to overcome hidden hunger. Plant Breeding 132, 437, 2013.

8. BHAGWAT S., GULATI D., SACHDEVA R., SANKAR R. Food fortification as a complementary strategy for the elimination of micronutrient deficiencies: case studies of large scale food fortification in two Indian States. Asia Pac. J. Clin. Nutr. 23, 4, 2014.

9. SPEROTTO R. A., RICACHENEVSKY F. K., WALDOW V. D. A., FETT J. P. Iron biofortification in rice: it's a long way to the top. Plant Sci. 190, 24, 2012.

10. KALAYCI M., ARISOY Z., ÇEKIÇ C., KAYA Y., SAVASLI E., TEZEL M., CAKMAK I. The effects of soil and foliar applications of zinc on grain zinc concentrations of wheat and maize. In Presentation presented at the Third International Zinc Symposium, Hyderabad, India. pp. 10-14, 2011.

11. CAKMAK I. Enrichment of cereal grains with zinc: agronomic or genetic biofortification? Plant Soil 302, 1, 2008.

12. LI B., ZHOU D., CANG L., ZHANG H., FAN X., QIN S. Soil micronutrient availability to crops as affected by longterm inorganic and organic fertilizer applications. Soil Till. Res. 96, 166, 2007.

13. FAROOQ M., WAHID A., SIDDIQUE K.H.M. Micronutrient application through seed treatments - a review. J. Soil Sci. Plant Nutr. 12, 125, 2012.

14. FOMINA M., GADD G. M. Biosorption: current perspectives on concept, definition and application. Bioresource Technol. 160, 3, 2014.

15. FU F., WANG Q. Removal of heavy metal ions from wastewaters: a review. J. Environ. Manage. 92, 407, 2011.

16. MUDHOO A., GARG V. K., WANG S. Removal of heavy metals by biosorption. Environ. Chem. Lett. 10, 109, 2012. 
17. GONZÁLEZ F., ROMERA E., BALLESTER A., BLÁZQUEZ M. L., MUÑOZ J. Á., GARCÍA-BALBOA C. Algal Biosorption and Biosorbents. In Microbial Biosorption of Metals (pp. 159-178). Springer Netherlands. 2011.

18. SAEID A., CHOJNACKA K., KORCZYŃSKI M., KORNIEWICZ D., DOBRZAŃSKI Z. Biomass of Spirulina maxima enriched by biosorption process as a new feed supplement for swine. J. Appl. Phycol. 25, 667, 2013.

19. WITKOWSKA Z., CHOJNACKA K., MICHALAK I. Application of Biosorption in the Production of Innovative Feed Supplements: A Novel Method. Adsorpt. Sci. Technol. 31, 421, 2013.

20. MICHALAK I., TUHY Ł., SAEID A., CHOJNACKA K. Bioavailability of $\mathrm{Zn}$ (II) to Plants from new Fertilizer Components Produced by Biosorption. Intl. J. Agron. Plant Prod. 4, 3522, 2013.

21. TUHY Ł., SAMORAJ M., MICHALAK I., CHOJNACKA $\mathrm{K}$. The Application of Biosorption for Production of
Micronutrient Fertilizers Based on Waste Biomass. Appl. Biochem. Biotechnology 174, 1376, 2014.

22. MICHALAK I., CHOJNACKA K. The new application of biosorption properties of Enteromorpha prolifera. Appl. Biochem. Biotechnology 160, 1540, 2010.

23. SWARAZ A. M., STANGOULIS J., KABIR A.H. Zincdeficiency resistance and biofortification in plants. J. Plant Nutr. Soil Sci. 177, 311, 2014.

24. HAFEEZ B., KHANIF Y. M., SALEEM M. Role of zinc in plant nutrition - a review. Am. J. Exp. Agric. 3, 374, 2013.

25. ALI M. M., AL-ANI A., EAMUS D., TAN D. K. Y. A New Image Processing Based Technique to Determine Chlorophyll in Plants. Am. Eurasian J. Agric. Environ. Sci. 12, 1323, 2012.

26. HU H., LIU H.Q., ZHANG H., ZHU J., YAO X., ZHANG X., ZHENG K. Assessment of chlorophyll content based on image colour analysis, comparison with SPAd-502. The 2 International Conference on Information Engineering and Computer Science Proceedings, Wuhan, China, 2010. 
